\title{
MINIMAX DESIGN OF CONSTRAINED PARABOLIC SYSTEMS*
}

\author{
Boris S. Mordukhovich \\ Department of Mathematics \\ Wayne State University \\ Detroit, MI 48202 \\ boris@math.wayne.edu
}

\begin{abstract}
This paper relates to minimax control design problems for a class of parabolic systems with nonregular boundary conditions and uncertain distributed perturbations under pointwise control and state constraints. The main attention is paid to the Dirichlet boundary control that offers the lowest regularity properties. Our variational analysis is based on well-posed multistep approximations and involves the solving of constrained optimal control problems for ODE and PDE systems. The design procedure essentially employs monotonicity properties of the parabolic dynamics and its asymptotics on the infinite horizon. Finally we justify a suboptimal three-positional structure of feedback boundary controllers and provide calculations of their optimal parameters that ensure the required system performance and robust stability under any admissible perturbations.
\end{abstract}

\section{INTRODUCTION}

In this paper we formulate and study a minimax feedback control problem for linear parabolic systems with uncertain disturbances and pointwise constraints on state and control variables. We deal with boundary controllers acting through the Dirichlet boundary conditions that are the most challenging for the parabolic dynamics. The original motivation comes from applications to some environmental problems; see [6] where a groundwater control problem of this kind has been considered for the case of one-dimensional heat-diffusion equations. Here we study a general class of multidimensional parabolic control systems that cover a fairly broad range of practical applications.

Dynamical processes in these systems are subject to pointwise (hard) state and control constraints that are typical in applied problems. Moreover, the

\footnotetext{
*This research was partly supported by the National Science Foundation under grant DMS9704751 and by the USA-Israel Binational Science Foundation under grant 94-00237.
}

The original version of this chapter was revised: The copyright line was incorrect. This has been corrected. The Erratum to this chapter is available at DOI: 10.1007/978-0-387-35359-3_40 
only information available for uncertain disturbances/perturbations is an admissible region of their variations. A natural approach to control design of such uncertain systems is minimax synthesis which guarantees the best system performance under the worst perturbations and ensures an acceptable behavior for any admissible perturbations. This approach is related to $H_{\infty}$-control and differential games; see, e.g., [1], [2], [3]. However, we are not familiar with any results in these theories that may be applied to parabolic systems with hard control and state constraints under consideration. In [4] the reader can find a number of feedback boundary control results for unconstrained parabolic systems based on Riccati equations.

In this paper we develop an efficient design procedure to solve minimax control problems for hard-constrained parabolic systems. This procedure takes into account monotonicity properties of the parabolic dynamics and asymptotic characteristics of transients on the infinite horizon. It was initiated in [6] for the case of one-dimensional heat-diffusion equations and then developed in [7] where partial results for multidimensional systems were reported. The results presented below include a justification of a suboptimal discontinuous feedback control structure and optimization of its parameters along the parabolic dynamics. In this way we minimize an energy-type cost functional in the case of maximal perturbations and ensure the desired state performance within the required constraints for all admissible disturbances. Based on a variational approach, we obtain verifiable conditions for "stability in the large" of the nonlinear closed-loop control system that excludes unacceptable self-vibrating regimes.

Our design and justification procedures involve multistep approximations and results from the optimal control theory for ordinary differential equations. As a by-product of this approach, we obtain a complete measure-free solution for a class of state-constrained optimal control problems related to approximations of the parabolic dynamics.

\section{PROBLEM FORMULATION AND BASIC PROPERTIES}

Consider a self-adjoint and uniformly strongly elliptic operator defined by

$$
A:=-\sum_{i, j=1}^{n} a_{i j}(x) \frac{\partial^{2}}{\partial x_{i} \partial x_{j}}-c
$$

where $c \in \mathbb{R}, a_{i j} \in C^{\infty}(\mathrm{cl} \Omega)$,

$$
\sum_{i, j=1}^{n} a_{i j}(x) \xi_{i} \xi_{j} \geq \nu \sum_{i=1}^{n} \xi_{i}^{2}, \nu>0 \forall x \in \Omega, \xi=\left(\xi_{1}, \xi_{2}, \ldots, \xi_{n}\right) \in \mathbb{R}^{n}
$$

$\Omega$ is a bounded open region in $\mathbb{R}^{n}$ with the sufficiently smooth boundary $\Gamma$. Given positive numbers $\underline{\alpha}, \bar{\alpha}, \underline{\beta}$, and $\bar{\beta}$, we define the sets of admissible controls $u(t)$ and admissible uncertain disturbances $w(t)$ by

$$
U_{a d}=\left\{u \in L^{2}(0, T) \mid u(t) \in[-\bar{\alpha}, \underline{\alpha}] \text { a.e. } t \in[0, T]\right\},
$$




$$
W_{a d}=\left\{w \in L^{2}(0, T) \mid w(t) \in[-\underline{\beta}, \bar{\beta}] \text { a.e. } t \in[0, T]\right\} .
$$

Suppose that $x_{0}$ is a given point in $\Omega$ at which we are able to collect information about the system performances, and let $\eta>0$. Consider the following minimax feedback control problem (P):

$$
\operatorname{minimize} J(u)=\max _{w(\cdot) \in W_{a d}} \int_{0}^{T}\left|u\left(y\left(t, x_{0}\right)\right)\right| d t
$$

over $u(\cdot) \in U_{a d}$ subject to the system

$$
\left\{\begin{array}{l}
\frac{\partial y}{\partial t}+A y=w(t) \text { a.e. in } Q:=(0, T) \times \Omega \\
y(0, x)=0, x \in \Omega \\
y(t, x)=u(t),(t, x) \in \Sigma:=(0, T] \times \Gamma,
\end{array}\right.
$$

the pointwise state constraints

$$
\left|y\left(t, x_{0}\right)\right| \leq \eta \forall t \in[0, T]
$$

and the feedback control law

$$
u(t)=u\left(y\left(t, x_{0}\right)\right)
$$

acting through the Dirichlet boundary conditions in (2).

Problem (P) formulated above is one of the most difficult control problems unsolved in the general theory. Our purpose is to develop an approach that takes into account specific features of parabolic systems and allows us to find a feasible suboptimal feedback control. To furnish this, we employ the spectral representation of solutions to the parabolic system (2) with the Dirichlet boundary conditions.

Let $\lambda \in \mathbb{R}$ be an eigenvalue of the operator $A$ in (1) and let $\phi \in L^{2}(\Omega)$ be the corresponding eigenfunction satisfying the condition $\left.\phi\right|_{\Gamma}=0$. It is well known that, under the assumptions made, one has the properties:

(a) All the eigenvalues $\lambda_{i}, i=1,2, \ldots$, of $A$ form a nondecreasing sequence that accumulates only at $\infty$, and the first eigenvalue $\lambda_{1}$ is simple.

(b) The corresponding orthonormal system of eigenfunctions is complete in $L^{2}(\Omega)$.

Let $y \in L^{2}(Q)$ be a generalized solution to (2) which uniquely exists for each $(u, w) \in U_{a d} \times W_{a d}$; see [5]. Based on the properties (a) and (b) and taking into account that both admissible controls and perturbations in (2) depend only on $t$, we conclude that the generalized solution $y(\cdot)$ admits the representation

$$
y(t, x)=\sum_{i=1}^{\infty} \mu_{i}\left(\int_{0}^{t} w(\theta) e^{\lambda_{i} \theta} d \theta+\left(c+\lambda_{i}\right) \int_{0}^{t} u(\theta) e^{\lambda_{i} \theta} d \theta\right) e^{-\lambda_{i} t} \phi_{i}(x)
$$


where $\mu_{i}=\int_{\Omega} \phi_{i}(x) d x$ and series (5) is strongly convergent in $L^{2}(Q)$. This allows us to deduce, involving the maximum principle for parabolic equations, the following monotonicity property of solutions to (2) with respect to both controls and perturbations.

Theorem 1. Let $\left(u_{i}, w_{i}\right) \in L^{2}(0, T) \times L^{2}(0, T)$ and let $y_{i}(\cdot), i=1,2$, be the corresponding generalized solutions to (2). Then

$$
y_{1}(t, x) \geq y_{2}(t, x) \text { a.e. in } Q
$$

if $u_{1}(t) \geq u_{2}(t)$ and $w_{1}(t) \geq w_{2}(t)$ a.e. in $[0, T]$.

One can see from Theorem 1 that the bigger magnitude of a perturbation is, the more control of the opposite sign should be applied to neutralize this perturbation and to keep the corresponding transient within the state constraint (3). This leads us to consider feedback control laws (4) satisfying the compensation property

$$
u(y) \leq u(\tilde{y}) \text { if } y \geq \tilde{y} \text { and } y \cdot u(y) \leq 0 \forall y, \tilde{y} \in \mathbb{R}
$$

The latter property implies that

$$
\int_{0}^{T}|u(y(t))| d t \geq \int_{0}^{T}|u(\tilde{y}(t))| d t \text { if } y(t) \geq \tilde{y}(t) \geq 0 \text { or } y(t) \leq \tilde{y}(t) \leq 0
$$

for all $t \in[0, T]$, i.e., the compensation of bigger (by magnitude) perturbations reguires more cost with respect to the maximized cost functional in $(\mathrm{P})$. This allows us to seek a suboptimal control structure in $(\mathrm{P})$ by examining the control response to feasible perturbations of the maximal magnitudes $w(t)=\bar{\beta}$ and $w(t)=-\underline{\beta}$ for all $t \in[0, T]$.

\section{SUBOPTIMAL CONTROL UNDER MAXIMAL PERTURBATIONS}

Taking into account the symmetry of $(\mathrm{P})$ relative to the origin, we consider the case of upper level maximal perturbations $w(\cdot)=\bar{\beta}$ and the corresponding set of admissible controls

$$
\bar{U}_{a d}:=\left\{u(\cdot) \in U_{a d} \mid-\bar{\alpha} \leq u(t) \leq 0 \text { a.e. } t \in[0, T]\right\} .
$$

To find an optimal control $\bar{u}(t)$ in respose to the maximal perturbations, we have the following open-loop control problem $(\overline{\mathrm{P}})$ :

$$
\operatorname{minimize} \bar{J}(u)=-\int_{0}^{T} u(t) d t
$$

over $u(\cdot) \in \bar{U}_{a d}$ subject to system (2) with $w(\cdot)=\bar{\beta}$ and the constraint

$$
y(t) \leq \eta \forall t \in[0, T]
$$


This is a state-constrained Dirichlet boundary control problem which was considered in [8] in more generality. In [8] we obtained necessary optimality conditions for $(\overline{\mathrm{P}})$ that involve the adjoint operator to the so-called Dirichlet map and Borel measures. Those conditions are rather complicated and do not allow us to determine an optimal control.

Following [6], let us explore another approach to solve problem $(\overline{\mathrm{P}})$. It leads to suboptimal feasible solutions of a simple structure that can be used to design and justify a required feedback law in the original minimax control problem (P). To furnish this, we approximate $(\overline{\mathrm{P}})$ by optimal control problems for ODE systems directly obtained from the spectral representation (5) as $x=x_{0}$ and $w(\cdot)=\bar{\beta}$. In what follows we suppose, additionally to the basic assumptions in Section 2, that the first eigenvalue $\lambda_{1}$ in (a) is positive. Together with the other properties in (a) this gives

$$
0<\lambda_{1}<\lambda_{i}, i=2,3, \ldots
$$

which ensures that the first term in (5) dominates as $t \rightarrow \infty$. This allows us to employ the first term rule [6] when the time interval is sufficiently large and thus to confine our treatment of suboptimality to the first order ODE approximation. In this way we arrive at the following constrained ODE optimal control problem $\left(\overline{\mathrm{P}}_{1}\right)$ : minimize the cost functional (7) along the controlled differential equation

$$
\dot{y}=-\lambda_{1} y+\mu_{1} \phi_{1}\left(x_{0}\right)\left(\bar{\beta}+\left(c+\lambda_{1}\right) u(t)\right) \text { a.e. } t \in[0, T], \quad y(0)=0
$$

subject to $u(\cdot) \in \bar{U}_{a d}$ and the state constraint (8).

The next theorem provides the complete exact solution of the state-constrained problem $\left(\overline{\mathrm{P}}_{1}\right)$ with no measure involved.

Theorem 2. Let $\mu_{1} \phi_{1}\left(x_{0}\right) \bar{\beta}>\lambda_{1} \eta$. Assume in addition that either

$$
\mu_{1} \phi_{1}\left(x_{0}\right)\left(\bar{\beta}-\bar{\alpha}\left(c+\lambda_{1}\right)\right) \leq \lambda_{1} \eta \text { or } \tau_{1}:=\frac{1}{\lambda_{1}} \ln \frac{\mu_{1} \phi_{1}\left(x_{0}\right) \bar{\beta}}{\mu_{1} \phi_{1}\left(x_{0}\right) \bar{\beta}-\lambda_{1} \eta} \geq T .
$$

Then system (8), (9) is controllable, i.e., there is $u(\cdot) \in \bar{U}_{a d}$ such that the corresponding trajectory of (9) satisfies the state constraint (8). Moreover, problem $\left(\overline{\mathrm{P}}_{1}\right)$ admits an optimal control of the form

$$
\bar{u}_{1}(t)= \begin{cases}0 & \text { if } t \in\left[0, \bar{\tau}_{1}\right) \\ \frac{\lambda_{1} \eta-\mu_{1} \phi_{1}\left(x_{0}\right) \bar{\beta}}{\mu_{1} \phi_{1}\left(x_{0}\right)\left(c+\lambda_{1}\right)} & \text { if } t \in\left[\bar{\tau}_{1}, T\right]\end{cases}
$$

where $\bar{\tau}_{1}=\min \left\{\tau_{1}, T\right\}$ with $\tau_{1}$ computed in $(10)$.

To prove this theorem, we first approximate $\left(\overline{\mathrm{P}}_{1}\right)$ by a parametric family of optimal control problems without state constraints. The latter problems can be completely solved by using the Pontryagin maximum principle [9] which provides necessary and sufficient conditions for optimality in this case. We prove 
that optimal controls to approximating problems are piecewise constant and contain both bang-bang and singular modes. Passing to the limit, we establish all the results of Theorem 2 and come to a surprising conclusion that the optimal control (11) for the state-constrained problem occurs to be simpler than the ones for the unconstrained approximations.

In the way we justify that a two-positional form in (11) can be accepted as a reasonable suboptimal control structure for problem $(\overline{\mathrm{P}})$ with the parabolic dynamics. Then we optimize this structure subject to (2), (8), and $u(\cdot) \in \bar{U}_{a d}$. This gives

$$
u(t)= \begin{cases}0 & \text { for } 0 \leq t<\tau \\ -\bar{u} & \text { for } \tau \leq t \leq T\end{cases}
$$

with the optimal parameters

$$
\bar{u}:=\frac{\gamma \bar{\beta}-\eta}{1+c \gamma}, \quad \gamma:=\sum_{i=1}^{\infty} \frac{\mu_{i} \phi_{i}\left(x_{0}\right)}{\lambda_{i}}
$$

and $\tau$ satisfying the equation

$$
\sum_{i=1}^{\infty} \frac{\mu_{i} \phi_{i}\left(x_{0}\right)}{\lambda_{i}} e^{-\lambda_{i} T}\left[\left(c+\lambda_{i}\right)(\gamma \bar{\beta}-\eta) e^{\lambda_{i} \tau}-\bar{\beta}(1+c \gamma)\right]=0
$$

We establish that (14) has a unique solution $\tau=\bar{\tau}(T) \in(0, T)$ for all $T$ sufficiently large and that any control $(12),(13)$ is feasible to $(\overline{\mathrm{P}})$ for all positive $\tau \leq \bar{\tau}(T)$. Moreover, the switching time $\tau=\bar{\tau}(T)$ is optimal in $(\overline{\mathrm{P}})$ and $\bar{\tau}(T) \downarrow \bar{\tau}$ as $\bar{T} \rightarrow \infty$ where the asymptotically optimal switching time $\bar{\tau}$ is computed by

$$
\bar{\tau}=\frac{1}{\lambda_{1}} \ln \frac{\bar{\beta}(1+c \gamma)}{\left(c+\lambda_{1}\right)(\gamma \bar{\beta}-\eta)} .
$$

\section{FEEDBACK CONTROL DESIGN}

The obtained results allow us to justify the three-positional control law

$$
u(y)= \begin{cases}-\bar{u} & \text { if } y \geq \bar{\sigma} \\ 0 & \text { if }-\underline{\sigma}<y<\bar{\sigma} \\ \underline{u} & \text { if } y \leq-\underline{\sigma}\end{cases}
$$

as a suboptimal feedback structure in (P) with the compensation property (6). Now using the monotonicity of transients with respect to both controls and perturbations as well as their asymptotic properties as $t \rightarrow \infty$, we arrive at the following theorem.

Theorem 3. Let the feedback control parameters $(\bar{u}, \bar{u}, \bar{\sigma}, \bar{\sigma})$ in (15) are computed by the formulas (13),

$$
\bar{\sigma}(T):=\bar{\beta}\left(\gamma-\sum_{i=1}^{\infty} \frac{\mu_{i} \phi_{i}\left(x_{0}\right)}{\lambda_{i}} e^{-\lambda_{i} \bar{\tau}(T)}\right),
$$


and their counterparts for $\underline{\beta}$. Then the control law (15) is feasible for any perturbations $w(\cdot) \in W_{\text {ad }}$ being optimal in the case of maximal perturbations when $T$ is sufficiently large. Moreover, $\bar{\sigma}(T) \downarrow \bar{\sigma}$ and $\underline{\sigma}(T) \downarrow \underline{\sigma}$ as $T \rightarrow \infty$ where the positive numbers

$$
\begin{gathered}
\bar{\sigma}:=\bar{\beta}\left(\gamma-\sum_{i=1}^{\infty} \frac{\mu_{i} \phi_{i}\left(x_{0}\right)}{\lambda_{i}}\left[\frac{\left(c+\lambda_{1}\right)(\gamma \bar{\beta}-\eta)}{\bar{\beta}(1+c \gamma)}\right]^{\frac{\lambda_{i}}{\lambda_{1}}}\right), \\
\underline{\sigma}:=\underline{\beta}\left(\gamma-\sum_{i=1}^{\infty} \frac{\mu_{i} \phi_{i}\left(x_{0}\right)}{\lambda_{i}}\left[\frac{\left(c+\lambda_{1}\right)(\gamma \underline{\beta}-\eta)}{\underline{\beta}(1+c \gamma)}\right]^{\frac{\lambda_{i}}{\lambda_{1}}}\right)
\end{gathered}
$$

form the maximal dead region $[-\underline{\sigma}, \bar{\sigma}]$ under which feedback (15) keeps the state constraints (3) on the infinite horizon $[0, \infty)$ for any admissible perturbations.

Finally we observe that the feedback control (15) with the parameters calculated in Theorem 3 does not guarantee the robust stability of the highly nonlinear (discontinuous) closed-loop system (2), (4), (15) under any admissible perturbations. Indeed, this system may have a self-vibrating regime (i.e., its zero-equilibrium is not stable in the large) if the dead region $[-\underline{\sigma}, \bar{\sigma}]$ is not sufficiently wide. The next theorem excludes such a possibility and ensures the required robust stability of the closed-loop control systems. Its proof is based on a variational approach and turnpike asymptotic properties of the parabolic dynamics.

Theorem 4. The closed-loop control system (2), (4), (15) with arbitrary parameters $(\bar{u}, \underline{u}, \bar{\sigma}, \underline{\sigma})$ is stable in the large if

$$
\bar{\sigma}+\underline{\sigma} \geq \min \{\bar{u}, \underline{u}\}\left[\frac{\mu_{1} \phi_{1}\left(x_{0}\right)\left(c+\lambda_{1}\right)}{\lambda_{1}}-(1+c \gamma)\right]>0 .
$$
form

When $\bar{\beta} \leq \underline{\beta}$, the stability condition (18) can be written in the simplified

$$
2 \bar{\sigma}_{1}+\underline{\sigma}_{1} \geq \eta
$$

through the suboptimal dead region bounds

$$
\begin{gathered}
\bar{\sigma}_{1}:=\bar{\beta}\left(\gamma-\frac{\mu_{1} \phi_{1}\left(x_{0}\right)\left(c+\lambda_{1}\right)(\gamma \bar{\beta}-\eta)}{\lambda_{1} \bar{\beta}(1+c \gamma)}\right), \\
\underline{\sigma}_{1}:=\underline{\beta}\left(\gamma-\frac{\mu_{1} \phi_{1}\left(x_{0}\right)\left(c+\lambda_{1}\right)(\gamma \underline{\beta}-\eta)}{\lambda_{1} \underline{\beta}(1+c \gamma)}\right)
\end{gathered}
$$

which correspond to the first terms in (16) and (17).

\section{References}

[1] Basar, T. and Bernard, P. (1995), $H_{\infty}$-Optimal Control and Related Minimax Design Problems, Birkhäuser, Boston. 
[2] van Keulen, B. (1993), $H_{\infty}$-Control for Distributed Parameter Systems: A State-Space Approach, Birkhäuser, Boston.

[3] Krasovskii, N.N. and Subbotin, A.I. (1988), Game-Theoretical Control Problems, Springer, New York, 1988.

[4] Lasiecka, I. and Triggiani, R. (1991) Differential and Algebraic Riccati Equations with Applications to Boundary/Point Control Problems, Springer, Berlin.

[5] Lions, J.L. (1971), Optimal Control of Systems Governed by Partial Differential Equations, Springer, Berlin.

[6] Mordukhovich, B.S. (1989) "Minimax design for a class of distributed parameter systems," Autom. Remote Control, Vol. 50, 262-283.

[7] Mordukhovich, B.S. and Zhang, K. (1996), "Feedback control design of constrained parabolic systems in uncertainty conditions," Prep. 13th IFAC World Congress, Vol. D, 225-230.

[8] Mordukhovich, B.S. and Zhang, K. (1997), "Minimax control of parabolic systems with Dirichlet boundary conditions and state constraints," Appl. Math. Optim., Vol. 36, 323-360.

[9] Pontryagin, L.S., Boltyanskii, V.G., Gamkrelidze, R.V. and Michshenko, E.F. (1962), The Mathematical Theory of Optimal Processes, WileyInterscience, New York. 